This item was submitted to Loughborough's Institutional Repository (https://dspace.lboro.ac.uk/) by the author and is made available under the following Creative Commons Licence conditions.

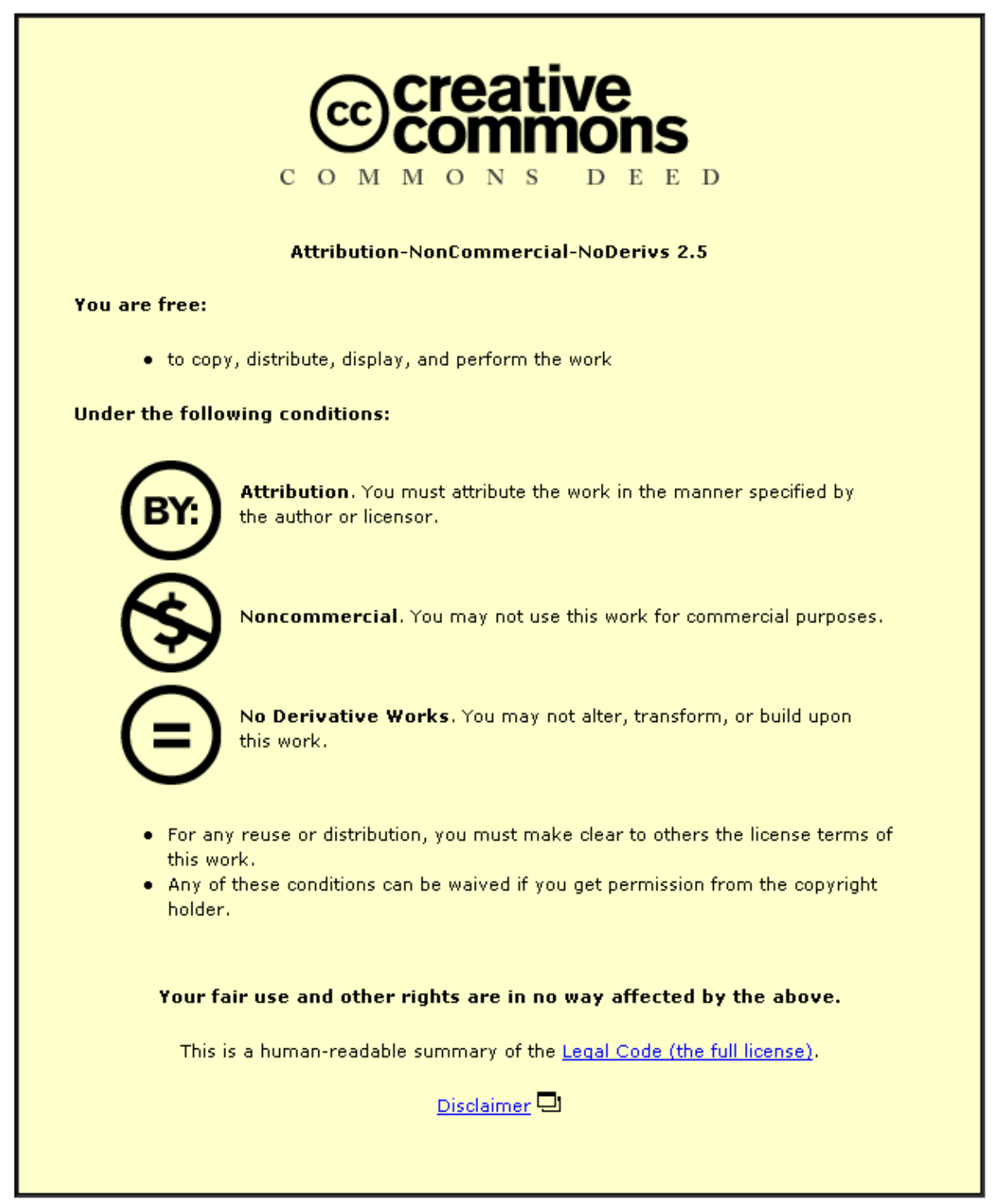

For the full text of this licence, please go to: http://creativecommons.org/licenses/by-nc-nd/2.5/ 


\title{
Nonthermal Atmospheric Plasmas Sustained Without Dielectric Barrier in the Kilohertz Range
}

\author{
J. J. Shi and M. G. Kong
}

\begin{abstract}
We report observation of nonthermal atmospheric discharges produced between two bare metallic electrodes over a wide frequency range from 20 to $260 \mathrm{kHz}$, in which generation of stable atmospheric glow discharges has so far necessitated dielectric barrier to be added to at least one electrode. Measured current and voltage characteristics suggest a distinctively different plasma-sustaining mechanism from that of atmospheric dielectric-barrier discharges. This is confirmed by hydrodynamic simulation.
\end{abstract}

Index Terms-Atmospheric pressure glow discharges, dielectric barrier, nonthermal plasma.

C URRENTLY, atmospheric pressure glow discharges (APGDs) are one of the most exciting areas in gas discharge research, because of their immense potentials for numerous applications, as well as the depth and scope of their scientific challenges [1]. Much attention has been paid to two broad groups of atmospheric glow discharges, namely atmospheric dielectric-barrier discharges (DBDs) generated between dielectrically insulated electrodes in the kilohertz range [2], [3] and radio-frequency APGD generated between bare electrodes in the megahertz range [4], [5]. Their generation and sustaining mechanisms are distinctively different. For atmospheric DBD, the dielectric barrier is considered to be essential to control the growth of discharge avalanche whereas this avalanche control in radio-frequency APGD is achieved by trapping electrons with a high excitation frequency [5].

In this paper, we report observation of stable and uniform atmospheric pressure glow discharges sustained between two bare metallic electrodes over a wide frequency range from 20 to $260 \mathrm{kHz}$. To our knowledge, this is the first evidence that dielectric coating of electrodes is no longer essential for APGD generation in the kilohertz range. In our experiments, these barrier-free APGD were generated in a helium flow at $5 \mathrm{slm}$ through a nominally $1.5-\mathrm{mm}$ gap between two parallel stainless steel electrodes. The top electrode is a round disk of $2 \mathrm{~cm}$ diameter and the bottom electrode is a rectangular plate of $5 \mathrm{~cm} \times 10 \mathrm{~cm}$. A low-frequency power supply is connected to the top electrode and delivers alternating high-voltage at a frequency between 20-260 kHz. Visual appearance of these barrier-free APGD is uniform without any apparent streamers. An example is given in Fig. 1.

Manuscript received June 21, 2004; revised September 20, 2004.

The authors are with the Department of Electronic and Electrical Engineering, Loughborough University, Leicestershire LE11 3TU, U.K. (e-mail: m.g.kong@lboro.ac.uk).

Digital Object Identifier 10.1109/TPS.2005.844997
Electrical measurements were made using voltage and current probes via a digital oscilloscope with a bandwidth of $300 \mathrm{MHz}$ and a sample rate of $2.5 \mathrm{Gs} / \mathrm{s}$. Measured current and voltage traces are repetitive and smooth, and no sharp spikes are present in the discharge current curve. It should be mentioned that the current probe is capable of resolving $2 \mathrm{~ns}$ and so can pick up sharp current spikes associated with filamentary discharges. The absence of any sharp current spikes on the measured current curve suggests that the generated barrier-free atmospheric plasma is a glow discharge. Typically the discharge current is less than $0.8 \mathrm{~mA}$, corresponding to a maximum current density of $0.25 \mathrm{~mA} / \mathrm{cm}^{2}$. Time-averaged dissipated power density calculated from measured current and voltage data is found to be below $1 \mathrm{~W} / \mathrm{cm}^{3}$, thus supporting further that the generated discharge is nonthermal plasma.

The discharge current of these barrier-free APGD exhibits a contrasting pattern from that in atmospheric DBD and radiofrequency APGD, particularly at low frequencies. Below 40 $\mathrm{kHz}$, the discharge current has a periodic pattern of one strong discharge event every half-cycle of the applied voltage. The strong discharge event occurs in the falling phase of the applied voltage. This is distinctively different from that of atmospheric DBD and radio-frequency APGD, for both of which a strong discharge event occurs in the voltage-rising phase in every half-cycle of the applied voltage. To confirm this difference, a one-dimensional hydrodynamic fluid model is developed based on our previous work [3]. Fig. 2 shows simulated and measured contour plots of the normalized discharge current over one and half cycles of the applied voltage at different excitation frequencies. In general both measured and simulated discharge currents exhibit a clear periodic pattern. The measured contour plot is less smooth because the applied voltage is not entirely sinusoidal and its waveform changes from one frequency to another. This is determined by the properties of the transformers of the power supply.

It is evident from Fig. 2 that there are two different discharge events and their relevant significance is dependent on the excitation frequency. At frequencies below $40 \mathrm{kHz}$, only one discharge event occurs in each half cycle and this is strongest at $t / T \sim 0.35>0.25$ in the voltage-falling phase. This is new and typical of barrier-free APGD at low frequencies. At an intermediate frequency between $40-60 \mathrm{kHz}$, there are two different discharge events every half-cycle with one in the voltage-falling phase and the additional one at $t / T=0.06-0.07$ in the voltagerising phase. They appear in an alternating fashion in time, and should influence the amount of residual electrons between two 


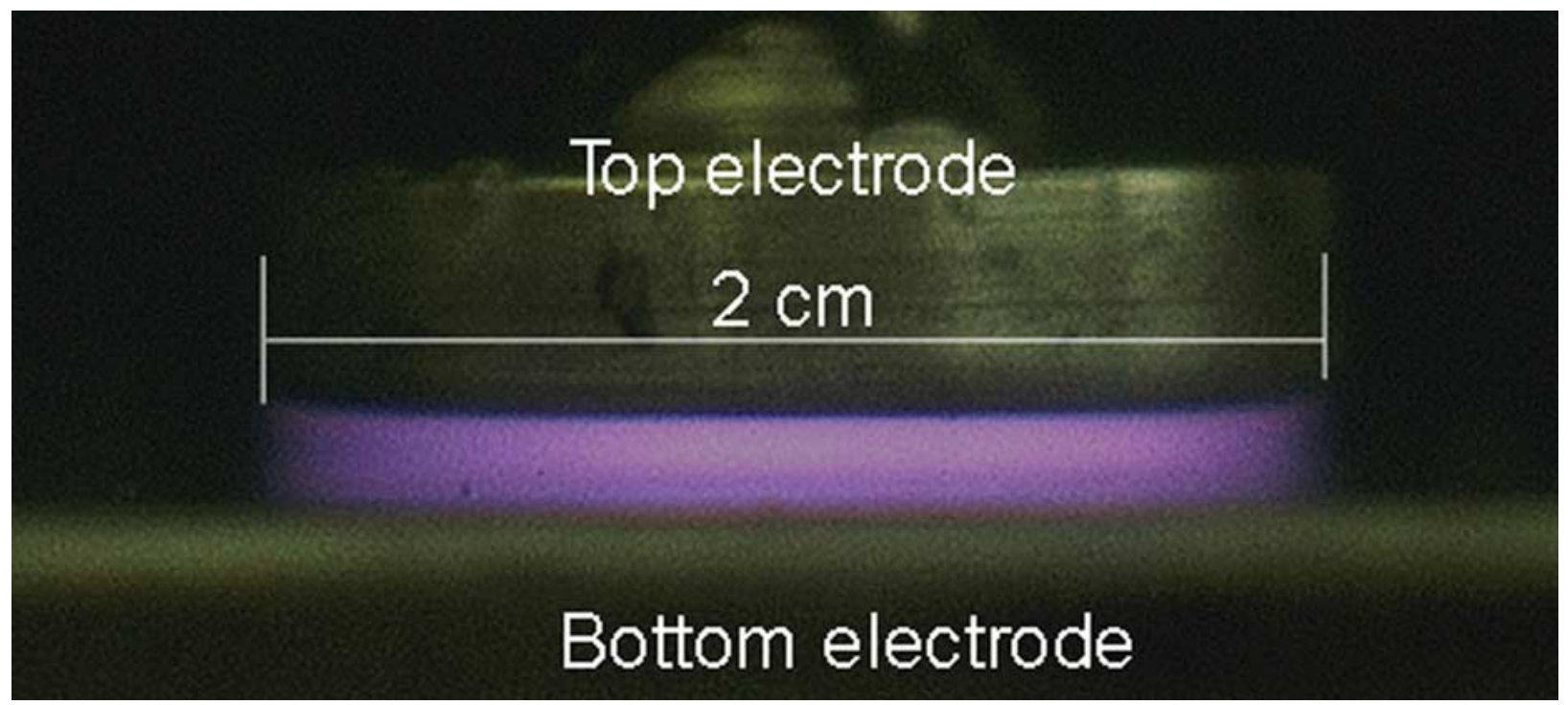

Fig. 1. Typical visual appearance of stable and uniform barrier-free atmospheric pressure glow discharges.
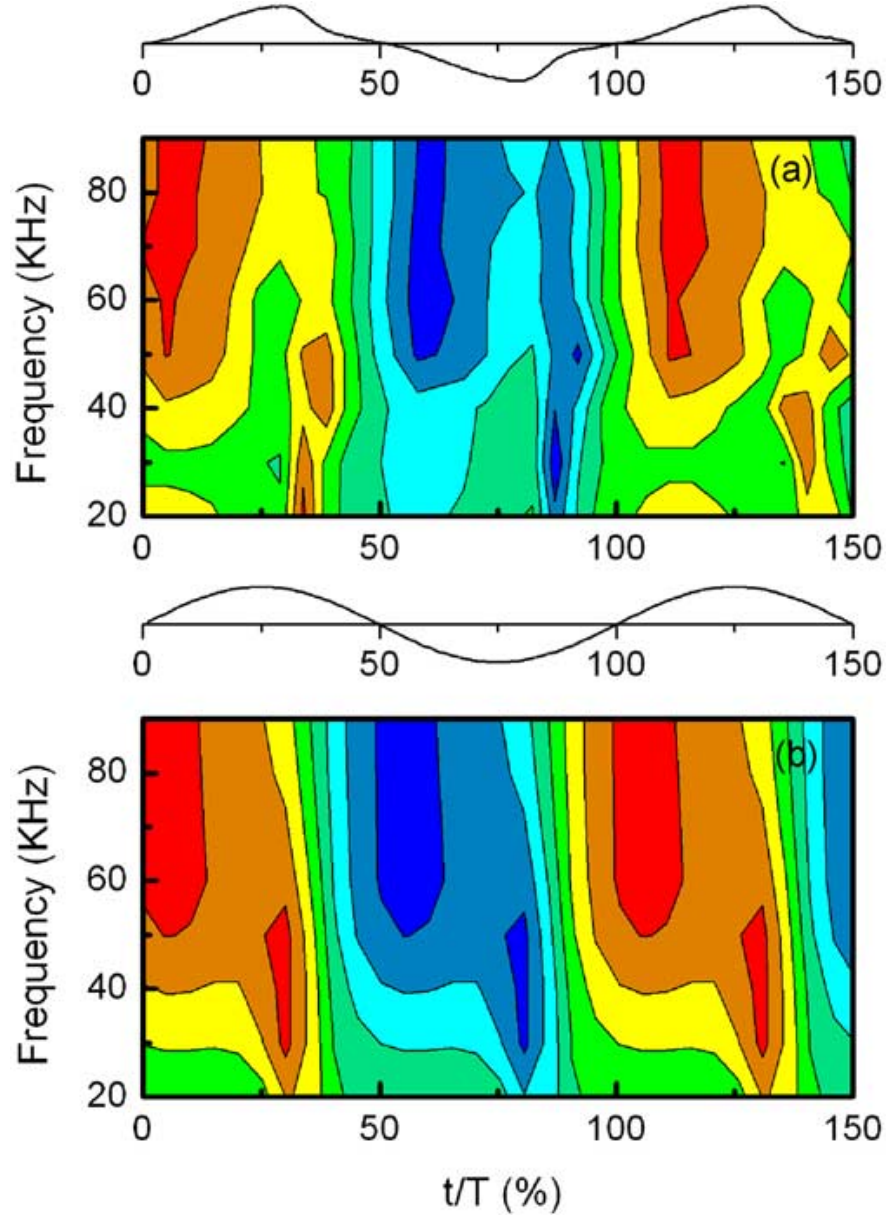

Fig. 2. Contour plots of temporal profile of (a) measured and (b) simulated discharge current normalized to its peak value at each frequency point from 20 to $90 \mathrm{kHz}$. Red color shows the positive maximum current and blue indicates the negative maximum current. Waveform of the applied voltage is shown on the top of each contour plot for 1.5 cycles.

consecutive discharge events. Therefore there is likely a competition between them. Over $60 \mathrm{kHz}$, the discharge event in the voltage-rising phase becomes dominant and that in the voltage-falling phase disappears. At $260 \mathrm{kHz}$, the discharge current pattern resembles closely that of radio-frequency APGD, both showing clear traits of capacitive discharges with the discharge current leading the voltage.

The coexistence of the two distinctively different discharge events observed between $40-60 \mathrm{kHz}$ is new and intriguing. It suggests that barrier-free APGD are sustained by two different mechanisms that are triggered in a sequential and alternating manner. Electron production and loss are therefore dynamically controlled, while the two different mechanisms interact with each other to maintain the generated barrier-free APGD. This intriguing phenomenon invites in-depth and thorough studies, which will be reported in a future note.

In summary, stable and uniform atmospheric pressure glow discharge were observed in a flowing helium gap between two parallel bare electrodes over a wide frequency range between 20-260 kHz. It has been shown that dielectric coating on the electrode surface is no longer necessary to obtain APGD in this frequency range. With the aid of a one-dimensional hydrodynamic fluid model, it has been established that at frequencies below $40 \mathrm{kHz}$ an hitherto unknown discharge event occurs in the voltage-falling phase while the usual discharge event in the voltage-rising phase becomes important at frequencies above $60 \mathrm{kHz}$. In the intermediate frequency range between 40-60 kHz, these two different discharge events coexist.

\section{REFERENCES}

[1] J. R. Roth, Industrial Plasma Engineering, Vol. I: Principles. Philadelphia, PA: Inst. Phys., 1995.

[2] S. Kanazawa, M. Kogoma, T. Moriwaki, and S. Okazaki, "Stable glow discharge at atmospheric pressure," J. Phys. D, Appl. Phys., vol. 21, pp. 838-840, May 1988

[3] M. G. Kong and X. T. Deng, "Electrically efficient production of a diffuse nonthermal atmospheric plasma," IEEE Trans. Plasma Sci., vol. 31 no. 1, pp. 7-18, Feb. 2003.

[4] A. Schutze, J. Y. Jeong, S. E. Babayan, J. Park, G. S. Selwyn, and R. F. Hicks, "The atmospheric-pressure plasma jet: A review and comparison to other plasma sources," IEEE Trans. Plasma Sci., vol. 26, no. 6, pp. 1685-1694, Dec. 1998.

[5] J. J. Shi, X. T. Deng, R. Hall, J. D. Punnett, and M. G. Kong, "Three modes in a radio frequency atmospheric pressure glow discharge," $J$ Appl. Phys., vol. 94, pp. 6303-6310, Nov. 2003. 